\title{
The Presence of Intra-lamina Lucida Blister Formation in Epidermolysis Bullosa Acquisita: Possible Role of Leukocytes
}

\author{
Jo-David Fine, M.D., Stephen Tyring, M.D., Ph.D., and W. Ray Gammon, M.D. \\ Department of Dermatology, University of Alabama at Birmingham; Birmingham Veterans Administration Medical Center, \\ Birmingham, Alabama; and Department of Dermatology, University of North Carolina, Chapel Hill, North Carolina, U.S.A.
}

In evaluating patients we have noted disparity between the locations of bound immunoreactants and the level of blistering in epidermolysis bullosa acquisita (EBA). We examined 10 consecutive EBA patients by routine histology, direct (DIF) and indirect (IIF; intact and $\mathrm{NaCl}$-split skin) immunofluorescence, immunofluorescence mapping (IM), and/or direct immunoelectron microscopy (DIEM). DIF was positive in each. IIF was positive in $3 / 8$ and $6 / 7$ patients when intact and split skin were used as substrates. DIEM revealed immunoreactants within the lamina densa (LD) in $6 / 10$, sub-LD in $1 / 10$, and both LD and sub-LD in $3 / 10$ patients. In contrast, by DIEM and IM, blister formation was noted within the lamina lucida (LL) in $7 / 9$ and $8 / 10$, sub-LD in $1 / 9$ and $1 / 10$, and within both LL and sub-LD in $1 / 9$ and
$1 / 10$, respectively. In the presence of neutrophils within the upper dermis $(n=6)$, cleavage occurred within the LL in 5 specimens; in one additional specimen containing predominantly neutrophils, cleavage occurred within both LL and sub-LD. In the presence of mononuclear cells $(n=2)$, intraLL cleavage occurred. In the presence of eosinophils, cleavage occurred within both LL and sub-LD. In the one specimen lacking any infiltrate, the cleavage plane was exclusively sub-LD. Intra-LL cleavage planes are more common than sub-LD ones in at least early cases of EBA. These findings likely represent the intra-LL-separating effect of leukocytederived proteolytic enzymes, when such cells are chemoattracted to the dermoepidermal junction by bound immunoreactants. J Invest Dermatol 92:27-32, 1989

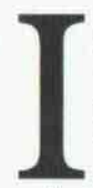

$\mathrm{n}$ recent years, several autoimmune dermatoses, including bullous pemphigoid, cicatricial pemphigoid, dermatitis herpetiformis (DH), linear IgA dermatosis, lupus erythematosus, and epidermolysis bullosa acquisita (EBA) have been more precisely characterized by a variety of immunologic techniques [1]. As a result, it is now apparent that many patients previously classified on the basis of clinical and direct immunofluorescence findings as having bullous pemphigoid instead have EBA [2].

It is generally accepted that the ultrastructural site of tissue-bound immunoreactants in most autoimmune diseases of the skin corre-

Manuscript received January 8, 1988; accepted for publication July 11, 1988.

Presented in part at the national meeting of the American Federation of Clinical Research, San Diego, CA, May 1987.

Supported by grants R23 AR34861 and 5R01 AR36629 from the National Institutes of Health and by research funding from the Medical Research Service of the Veterans Administration

Reprint requests to: Jo-David Fine, M.D., Department of Dermatology, University of Alabama at Birmingham, University Station, Box 76, Birmingham, Alabama 35294

Abbreviations:

DH: dermatitis herpetiformis

DIEM: direct immunoelectron microscopy

DIF: direct immunofluorescence

EBA: epidermolysis bullosa acquisita

FITC: fluorescein isothiocyanate

IIF: indirect immunofluorescence

IM: immunofluorescence mapping

LD: lamina densa

LL: lamina lucida lates with the level of blister formation (i.e., both reaction products and cleft formation within the lamina lucida) [1]. In the case of DH, however, tissue-bound immunoreactants have been localized to the sub-lamina densa region of the basement membrane zone [3-5], whereas, more recently, skin cleavage has been shown to occur within the lamina lucida itself $[6,7]$. This disparity has been ascribed to an inherent weakness ("locus resistensia minoris") of the lamina lucida [6]. In the course of studies of patients with several of these autoimmune diseases, we have noted a similar disparity in skin specimens from patients with proven EBA. We now report on our findings in ten consecutive patients with EBA and propose an alternate explanation for the findings most commonly seen in both $\mathrm{DH}$ and EBA.

\section{MATERIALS AND METHODS}

Patients Examined 10 patients ( 4 male, 6 female), with an average age of 62 (range, $41-82$ ), subsequently shown to have EBA, were included. A summary of their clinical findings is listed in Table I. The diagnosis of EBA was considered in those bullous disease patients meeting the following minimal criteria: positive direct immunofluorescence for linear homogeneous immunoreactant(s) (other than only IgA) along the dermoepidermal junction, demonstration of tissue-bound immunoreactants within and/or below the lamina densa (by direct immunoelectron microscopy), and lack of clinical and serologic findings suggestive of systemic lupus erythematosus [8-10]. When possible, indirect immunofluorescence on intact and $\mathrm{NaCl}$-split skin was also performed; when the latter study was positive, the diagnosis of EBA was further confirmed by the detection of anti-basement membrane autoantibody staining solely upon the dermal base of such intra-lamina lucida separated skin [11]. 
Table I. Summary of Clinical Findings of 10 Patients with EBA

\begin{tabular}{|c|c|c|c|c|c|}
\hline Patient & $\mathrm{Age}^{2}$ & Sex & Symptoms Duration ${ }^{b}$ & Pruritus & Burning \\
\hline 1 & 59 & $\mathrm{M}$ & $8 \mathrm{mo}$. & + & + \\
\hline 2 & 69 & $\mathrm{~F}$ & 1 day & + & + \\
\hline 3 & 56 & $\mathrm{~F}$ & $3 \mathrm{mo}$. & + & + \\
\hline 4 & 62 & $\mathrm{~F}$ & $3 \mathrm{mo}$. & + & + \\
\hline 5 & 81 & $\mathrm{M}$ & $4 \mathrm{mo}$ & + & - \\
\hline 6 & 47 & $\mathrm{M}$ & $18 \mathrm{mo}$. & - & - \\
\hline 7 & 64 & F & $6 \mathrm{yr}$. & - & - \\
\hline 8 & 82 & F & $6 \mathrm{mo}$ & + & - \\
\hline 9 & 41 & F & $6 \mathrm{yr}$. & + & + \\
\hline \multirow[t]{2}{*}{10} & 54 & M & $3 \mathrm{yr}$. & + & + \\
\hline & & & & 8 & 6 \\
\hline
\end{tabular}

Lesional Morphology

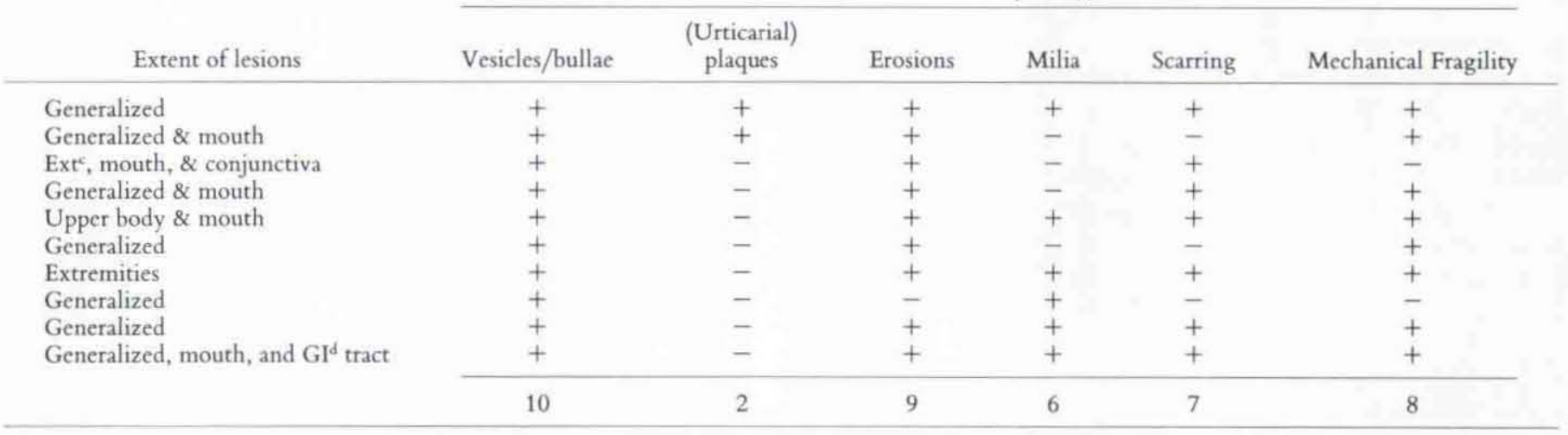

At time of diagnosis.

b Prior to definitive diagnosis.

Ext: extremities.

d GI: gastrointestinal.

Routine Histology 4-mm punch biopsies were obtained from lesional skin in 10 patients. Each specimen was stained with hematoxylin and eosin and examined for both the level of skin cleavage (intraepidermal versus subepidermal) and the type(s) of inflammatory cell(s) present within the blister cavity and within the dermal perivascular infiltrate.

Direct Immunofluorescence (DIF) Studies DIF was performed on perilesional skin $(n=10)$, lesional skin $(n=1)$, and normal-appearing buccal mucosa $(\mathrm{n}=1)$ by a standard technique utilizing FITC-conjugated goat antihuman antibodies to IgG, IgA, $\mathrm{IgM}, \mathrm{IgE}$, and fibrinogen (Cappel Laboratories, Cochranville, PA).

Indirect Immunofluorescence (IIF) Studies IIF was performed on both intact normal human skin $(n=8)$ (both adult skin and neonatal foreskin) and $\mathrm{NaCl}$-split human skin $(\mathrm{n}=7)$, the latter as previously reported [11]. In brief, the latter tissue substrate was prepared by first subjecting intact human skin to $1.0 \mathrm{M} \mathrm{NaCl}$ solution for $72 \mathrm{~h}$ at $4^{\circ} \mathrm{C}$, following which the epidermis was carefully removed mechanically from the underlying dermis, and both specimens snap frozen in O.C.T. embedding medium (Lab-Tek Products, Napersville, IL), and stored at $-70^{\circ} \mathrm{C}$ until needed. Eight-micron-thick cryostat sections were prepared from each frozen specimen and a two-step IIF procedure was performed on each of the two human skin substrate preparations utilizing undiluted and serially diluted serum from each EBA patient (in two-fold dilutions through $1: 32$, in $0.0067 \mathrm{M}$ phosphate buffered saline ( $\mathrm{pH} 7.4$ ). Following incubation at room temperature in a humidified chamber for $30 \mathrm{~min}$, each specimen was briefly rinsed in PBS, further incubated for $30 \mathrm{~min}$ in the presence of FITC-conjugated goat antihuman IgG (1: 40 dilution; Cappel Laboratories), further rinsed in PBS, and then examined by epi-illumination with a Leitz Laborlux 12 immunofluorescence microscope, following the mounting of each tissue-containing slide with $50 \%$ glycerol in PBS. Controls for the split skin studies included previously documented bullous pemphigoid antisera (positive controls) and normal human sera (negative controls).

Immunofluorescence Mapping (IM) Studies Immunofluorescence mapping $[12,13]$ was performed on 11 specimens previously examined by DIF technique. These specialized indirect immunofluorescence studies were performed utilizing well-characterized polyclonal antibodies to the following skin basement membrane antigens: bullous pemphigoid antigen, laminin, and type IV collagen. As controls, we utilized normal sera from the appropriately matched species (human, sheep). These mapping studies were performed as previously described over a range of antibody titers (anti-bullous pemphigoid antigen, $1: 10-1: 20$; anti-laminin, and anti-type IV collagen, $1: 40-1: 1280$ ) known to result in strong positive staining on normal intact and $\mathrm{NaCl}$-separated human skin and on skin specimens from patients with each of the three forms of inherited epidermolysis bullosa. FITC-conjugated goat anti-human IgG $(1: 40)$ and rabbit anti-sheep IgG (1:20) (Cappel Laboratories) were employed as second step immunoreagents. Incubation times and temperatures were identical to those used for split-skin studies. Determination of the site(s) of in vivo bound immunoreactants was accomplished by examining such tissues following incubation with normal human serum $(1: 10-1: 20)$. Subsequently, the localization of each of the three above-mentioned antigens, and therefore the level of skin cleavage in each specimen, was determined by comparing the staining of basement membrane with each of these polyclonal antibodies and with normal human serum. Specifically, intra-lamina lucida cleavage was characterized by the presence of bullous pemphigoid antigen on the roof and laminin and type IV collagen along the base of the blister, whereas in sub-lamina densa cleavage all three antigens were noted along the blister roof, consistent with the ultrastructural localization of each of these antigens in normal intact human skin. 
Direct Immunoelectron Microscopic (IEM) Studies Direct IEM was performed on 11 specimens previously examined by DIF technique, using methodology previously described $[14,15]$. In brief, $10-\mu \mathrm{m}$-thick cryostat sections of unfixed human skin or mucosa were prepared, and serial incubations (room temperature, 30 $\mathrm{min}$ ) were carried out with the following sequence of immunoreagents: normal rabbit serum (to block nonspecific binding of later steps), goat antihuman immunoglobulin or $\mathrm{C} 3$ (the specific one chosen dependent on the DIF findings in each specimen), rabbit antigoat IgG, goat peroxidase-anti-peroxidase, and a Tris buffer solution ( $\mathrm{pH} 7.6$ ) containing diaminobenzidine and hydrogen peroxide. Following extensive washing in PBS, each specimen was then further prepared for evaluation by transmission electron microscopy as previously described $[14,15]$.

\section{RESULTS}

Clinical findings on the 10 patients included in this study are summarized in Table I. In seven patients, the eruption was generalized; mucosal involvement was noted in five. The most common clinical findings included vesicle or bulla formation $(10 / 10)$, erosions $(9 / 10)$, mechanical fragility $(8 / 10)$, and scar formation $(7 / 10)$. Less frequently, milia and urticarial plaques were also observed. Most patients were symptomatic for either pruritus $(8 / 10)$ or a marked burning sensation of the skin $(6 / 10)$.

Direct and indirect immunofluorescence findings and those of routine histology are depicted in Table II. Twelve tissue specimens were obtained from these patients. In all but two specimens, the biopsies were from perilesional skin. The most common tissuebound immunoreactants were IgG and C3, each of which was noted in $9 / 10$ patients (11/12 specimens). Less frequently, fibrinogen, $\operatorname{IgA}$, IgM, and in one patient, $\operatorname{IgE}$, were also detected. When compared to specimens from patients with bullous pemphigoid, in which only two immunoreactants, IgG and C3, are usually detectable along the dermoepidermal junction [1], 7/10 EBA patients had at least three in vivo bound immunoreactants present along the dermoepidermal junction.

Similar to that previously reported in EBA $[2,10]$, slightly less than $40 \%$ of our subjects had detectable anti-basement membrane autoantibodies when intact human skin was used as the tissue substrate. In contrast, $6 / 7$ of the sera examined had detectable antibasement membrane autoantibodies when $\mathrm{NaCl}$-split human skin was utilized as substrate. Identical to that previously published in EBA [11], these autoantibodies bound solely to the dermal portion of the intra-lamina lucida separated skin. In contrast, control anti-
Table III. Direct Immunoelectron Microscopic (IEM) and Immunofluorescence Mapping (IM) Findings

\begin{tabular}{|c|c|c|c|c|}
\hline \multirow[b]{2}{*}{ Patient } & \multicolumn{2}{|c|}{ IEM } & \multicolumn{2}{|c|}{ IM } \\
\hline & Bound-IR ${ }^{b}$ & Cleft(s) & Bound-IR ${ }^{b}$ & Cleft(s) \\
\hline 1 & $\mathrm{LD}^{2}$ & LL $^{c}$ & Base & LL \\
\hline 2 & LD & LL & Base & LL \\
\hline 3 & $\mathrm{LD}^{\mathrm{d}}$ & LL & Base \& roof ${ }^{2}$ & $\mathrm{LL}^{2}$ \\
\hline 4 & LD, sub-LD & LL, sub-LD & Base & LL \\
\hline 5 & LD & LL & Base & LL \\
\hline 6 & LD, sub-LD & LL & Base & LL \\
\hline 7 & Sub-LD & Sub-LD & Roof & Sub-LD \\
\hline 8 & LD, sub-LD & LL & Base & LL, sub-LD \\
\hline 9 & LD & LL & Base & LL \\
\hline 10 & LD & $(-)^{e}$ & Base & LL \\
\hline
\end{tabular}

- On each of 2 specimens.

bIR: immunoreactants.

LL: lamina lucida.

¿LD: lamina densa.

e (-): not visualized.

sera from several patients with bullous pemphigoid bound exclusively to the undersurface of the separated epidermal roof (data not shown).

As noted in Table II, some inflammatory infiltrate was noted in all but one specimen. The most common type of inflammatory cell detected was the polymorphonuclear leukocyte $(6 / 10)$. Less commonly, mononuclear cells $(2 / 10)$ or eosinophils $(1 / 10)$ predominated.

Direct IEM and IM were performed in 11 specimens from these 10 EBA patients; results are depicted in Table III. In 9/11 specimens, a single cleavage plane was detectable. In two specimens, a second and more minor cleavage plane was also focally present. With the few exceptions noted in Table III, there was consistency in the findings observed by both direct IEM and IM techniques. In $10 / 11$ specimens, tissue-bound immunoreactants were noted by IM exclusively on one side of the blister ( 9 dermal base, 1 epidermal roof), corresponding to those findings noted by direct IEM (Fig $1 A-D)$. In one specimen, both epidermal roof and dermal base contained tissue-bound immune deposits. By direct IEM, all immunoreactants were shown to reside within the lamina densa, sublamina densa, or both lamina densa and sub-lamina densa regions in 7,1 , and 3 specimens, respectively, consistent with previously pub-

Table II. Routine Histologic and Direct and Indirect Immunofluorescence Findings in EBA

\begin{tabular}{|c|c|c|c|c|c|c|c|c|c|c|}
\hline \multirow[b]{2}{*}{$\mathrm{Pt}$} & \multirow[b]{2}{*}{ Site $^{2}$} & \multirow[b]{2}{*}{ Cellular Infiltrate } & \multicolumn{6}{|c|}{ DIF $^{b}$} & \multicolumn{2}{|c|}{$\operatorname{IIF}^{c}$ (on skin) } \\
\hline & & & $\operatorname{IgG}$ & $\operatorname{IgA}$ & $\operatorname{Ig} M$ & $\mathrm{IgE}$ & C3 & Fibrinogen & Intact & Split \\
\hline \multirow[t]{2}{*}{1} & Lesional & $\mathrm{PMN}^{\mathrm{d}}$ & + & - & + & - & + & + & - & + \\
\hline & Perilesional & PMN & + & + & - & - & + & + & & \\
\hline 2 & Perilesional & PMN & + & + & - & - & + & + & - & + \\
\hline \multirow[t]{2}{*}{3} & Perilesional & Mono ${ }^{e}$ & + & + & + & - & + & - & + & + \\
\hline & Normal buccal mucosa & Mono & + & - & - & - & + & + & & \\
\hline 4 & Perilesional & PMN & + & - & - & - & + & - & - & + \\
\hline 5 & Perilesional & PMN & + & - & - & - & + & + & - & - \\
\hline 6 & Perilesional & Mono & - & + & + & - & + & + & & \\
\hline 7 & Perilesional & None & + & - & + & - & - & - & - & \\
\hline 8 & Perilesional & $\operatorname{Eos}^{f}$ & + & + & - & - & + & + & & \\
\hline 9 & Perilesional & PMN & + & - & - & - & + & - & + & + \\
\hline 10 & Perilesional & PMN & + & - & - & + & + & - & + & + \\
\hline \multicolumn{2}{|c|}{$\begin{array}{l}\mathrm{n}=\text { positive patients } \\
\quad \text { (total }=10 \text { unless noted) }\end{array}$} & & 9 & 5 & 4 & 1 & 9 & 6 & $3 / 8$ & $6 / 7$ \\
\hline
\end{tabular}

- Skin except where noted.

bIF: direct immunofluorescence.

IIF: indirect immunofluorescence.

¿ PMN: polymorphonuclear leukocytes.

- Mono: mononuclear leukocytes.

Eos: cosinophils. 

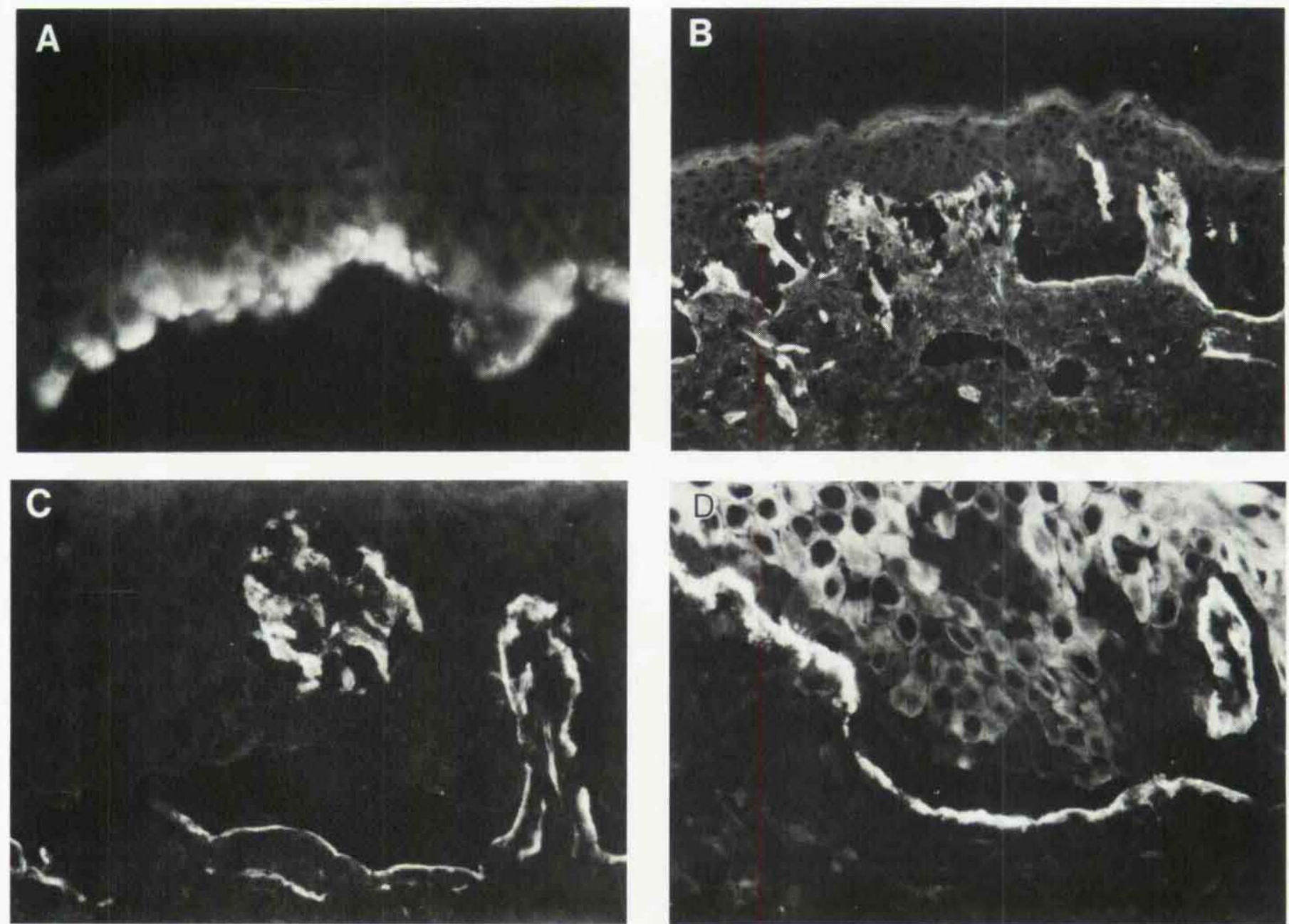

Figure 1. Immunofluorescence mapping study on perilesional EBA skin, using antibodies to bullous pemphigoid antigen $(A)$, laminin (B), type IV collagen $(C)$, and normal human serum $(D)$. The distribution of antibody binding in $A-C$ is indicative of intra-lamina lucida cleavage, whereas staining in $D$ demonstrates the localization of in vivo bound immunoreactants to be within or beneath the level of the lamina densa. ( $A: 550 \mathrm{x} ; B: 138 \mathrm{x} ; C$ and $D: 344 \mathrm{x}$ ).

lished data on skin from patients with EBA [2,8-11], as depicted in Figs 2 and 3 . Similar localization of tissue-bound immunoreactants was deduced from IM findings.

The location of cleavage plane(s) detectable by IM and direct IEM was compared to the predominant type of inflammatory cell within the infiltrate. Although none of the EBA tissue specimens contained tissue-bound immunoreactants within the lamina lucida, exclusive intra-lamina lucida cleavage was noted in $7 / 10$ specimens (Fig 1 3). In five of these seven patients, the primary dermal inflammatory cell was the polymorphonuclear leukocyte; in two, mononuclear cells were noted. In two additional patients, a second and smaller cleft was noted in the sub-lamina densa region; in these patients, polymorphonuclear leukocytes or eosinophils were noted. In contrast, in the one patient having exclusively sub-lamina densa blister formation, no inflammatory cellular infiltrate was noted.

\section{DISCUSSION}

Several autoimmune skin diseases, including bullous pemphigoid, cicatricial pemphigoid, and herpes gestationis, have both blister formation and tissue-bound immunoreactants within the lamina lucida $[1,16,17]$. In contrast, in two autoimmune bullous diseases, epidermolysis bullosa acquisita [2,8-11] and bullous eruption of systemic lupus erythematosus [18-20], tissue-bound immunoreactants have been shown to reside with the lamina densa and/or sublamina densa regions. In addition, it has been reported that blister formation in both conditions occurs beneath the region of the lamina densa $[8,9,18-20]$.

Based upon our experience with 10 consecutive patients subse- quently shown to have EBA by direct IEM and $\mathrm{NaCl}$-split skin studies, the presence of three or more tissue-bound immunoreactants along the dermoepidermal junction in a linear homogeneous pattern appears to be an early clue to the diagnosis of EBA; similar direct immunofluorescence findings have been previously noted by others in EBA [21] and also have been reported recently in cicatricial pemphigoid [15]. As previously reported, antiskin basement membrane autoantibodies were seen in the majority of our EBA patients when $\mathrm{NaCl}$-split skin was used as the tissue substrate for IIF, whereas such autoantibodies were detectable in only about $40 \%$ of patients when intact human skin was employed. The high frequency of antiskin basement membrane autoantibodies detectable in EBA and their exclusive binding to the dermal portion of $\mathrm{NaCl}-$ split skin further confirms the usefulness of this latter substrate in both diagnosing EBA and readily differentiating this entity from bullous pemphigoid.

In contrast to previous reports, however, a majority of specimens from our series of EBA patients were noted to have intra-lamina lucida skin cleavage rather than sub-lamina densa clefts. Infrequently, both intra-lamina lucida and sub-lamina densa clefts or exclusively sub-lamina densa clefts were noted. These findings may be contrasted to the presence of lamina densa and/or sub-lamina densa-bound immunoreactants in all patients that we have examined with EBA.

When the localization of skin cleavage was compared to the findings seen on routine histology, there appears to be a correlation between the presence of leukocytes within the upper dermis directly underneath the dermo-epidermal junction and the presence of 
Figure 2. Direct immunoelectron microscopy of perilesional skin from patient 2, demonstrating tissue-bound immunoreactants within and beneath the lamina densa (solid arrowheads) and early blister formation within the lamina lucida (solid arrow). (magnification: 25,000x).

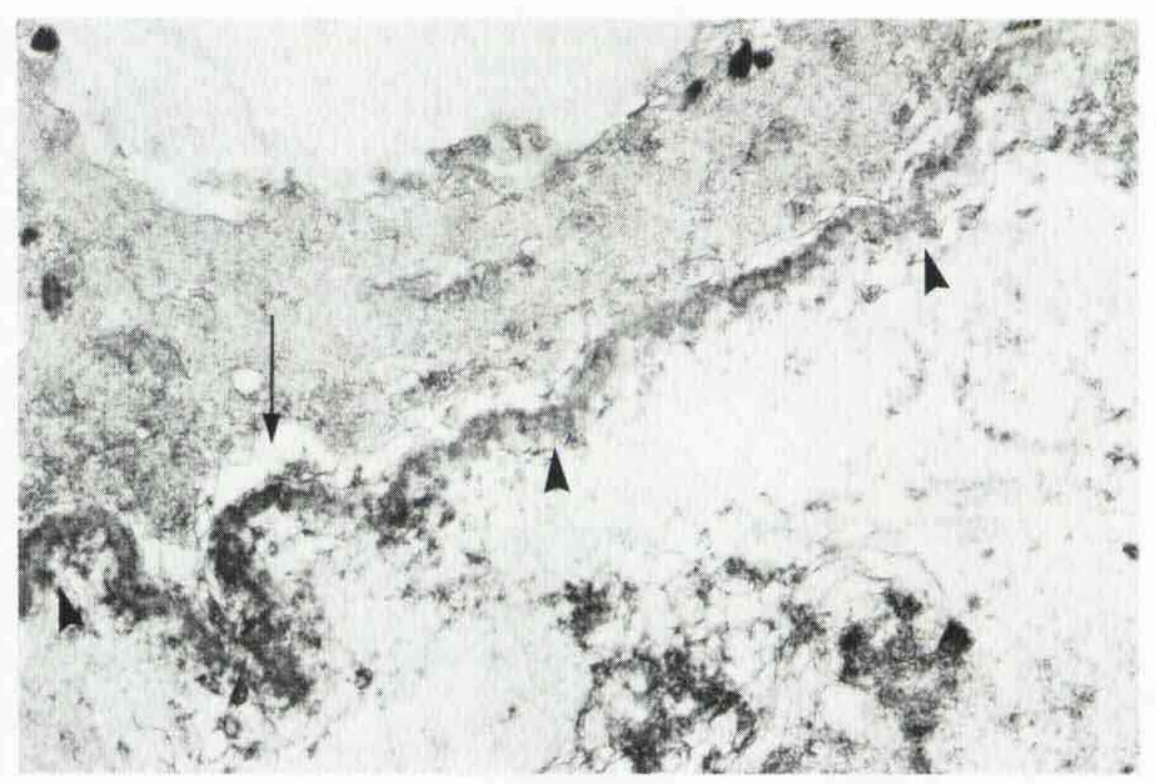

intra-lamina lucida cleavage. That is, a predominant or exclusive intra-lamina lucida cleavage plane was noted in each of the nine EBA patients having leukocytic infiltrates within the upper dermis adjacent to the dermo-epidermal junction. These latter findings may be analogous to those reported in dermatitis herpetiformis, in which a neutrophilic infiltrate is present within the upper papillary dermis, tissue-bound immunoreactants are localized to the sub-lamina densa region, and blister formation may occur within the lamina lucida [3 - 7]. In contrast, only sub-lamina densa cleavage was detectable in the one patient lacking an inflammatory dermal infiltrate, further suggesting the possibility of a causative role for leukocytes in the development of intra-lamina lucida separation in EBA. Despite this latter observation, however, it is clear that further critical evaluation of a large series of specimens from EBA patients lacking apparent dermal inflammation will be necessary to substantiate the validity of this hypothesis.

Recent experiments have demonstrated that normal human leukocytes, particularly polymorphonuclear leukocytes, are capable of releasing enzymes which, when incubated in vitro with sections of intact human skin, lead to intra-lamina lucida separation and subsequent dissolution of the basement membrane zone [22]. These latter findings suggest a model which appears to explain the apparent disparity between the level of blister formation and that of tissuebound immunoreactants in both dermatitis herpetiformis and EBA. It is possible that cleavage in DH occurs within the lamina lucida because of the activity of proteolytic enzymes that have been released by leukocytes chemoattracted to the dermo-epidermal junction by tissue-bound IgA in that vicinity; in this manner, enzymatic degradation of one or more components within the lamina lucida would lead to eventual cleft formation at that location. Similarly, it is likely, at least in early cases of EBA, that the presence of tissuebound immunoreactants within the lamina densa and/or sub-lamina densa may result in the chemoattraction to the dermo-epidermal junction of one or more types of leukocytes which subsequently release enzymes leading to intra-lamina lucida blister formation $[23]$. Because some patients with EBA and neutrophilic inflammatory infiltrates do have sub-lamina densa cleavage, however, it is obvious that other factors, possibly including the duration of disease, the level or extent of disease activity, and/or additional mechanical instability of the dermo-epidermal junction due to alterations in anchoring fibrils, microfibrillar bundles, collagen, and/or elastic fibers [24], and the quantity and extent of tissue-bound immunoreactants within the sub-lamina densa region in some patients may also contribute to deeper levels of skin cleavage and blister formation than that observed in our present series of patients. For example, one reasonable explanation for the disparity in findings between our patient population and those previously described is the rather short duration of disease in the former group. As a result of our prospectively evaluating all newly diagnosed patients with autoimmune bullous dermatoses by direct IEM during the course of this study, we were able to study most EBA patients during the earliest course (frequently referred to as the inflammatory stage) of their disease; in six of the nine with inflammatory infiltrates, the average onset of disease was only 6 months prior to our evaluation. As previously noted, the skin biopsy from one of our patients (no. 7) lacked any evidence of inflammatory infiltrate; this same patient was the only one noted to have exclusively sub-lamina densa blister formation. Although it is unknown why such deep cleavage occurred in this patient, a few possible explanations can be suggested. First, the duration of disease activity in this patient was at least 6 years. As such, the chronicity of disease in this particular patient may have allowed for extensive damage to the upper dermal structures, including anchoring fibrils, thereby predisposing the skin to sub-lamina densa cleavage. Second, longstanding deposition of immunoreactants within the sub-lamina densa may have further compromised the mechanical integrity of this region. Third, in the absence of leukocytes and their enzymatic products, there was no additional complicating tissue injury favoring intra-lamina lucida rather than sub-lamina densa blister formation.

As a practical point, the diagnosis of EBA cannot be excluded on the basis of the demonstration of intra-lamina lucida cleavage by either routine transmission electron microscopy or immunofluorescence mapping techniques unless concurrent direct IEM demonstrates that these immunoreactants are present within (i.e., as in bullous pemphigoid) rather than beneath the lamina lucida. Furthermore, this may explain the findings in one recent study of bullous pemphigoid in which some patients, in part defined as having bullous pemphigoid by the presence of intra-lamina lucida cleavage, were noted to have immunoreaction products beneath that ultrastructural region [25]; presumably those patients in fact had EBA with clinical features mimicking those of bullous pemphigoid [2]. Considering the frequent clinical similarity between bullous pemphigoid and EBA, the definitive differentiation of these two entities should rest upon the findings of at least one of two specialized techniques: direct IEM or, if available and positive, the results of immunoblot analysis of circulating autoantibodies when the latter are exposed to extracted bullous pemphigoid and EBA antigens. 


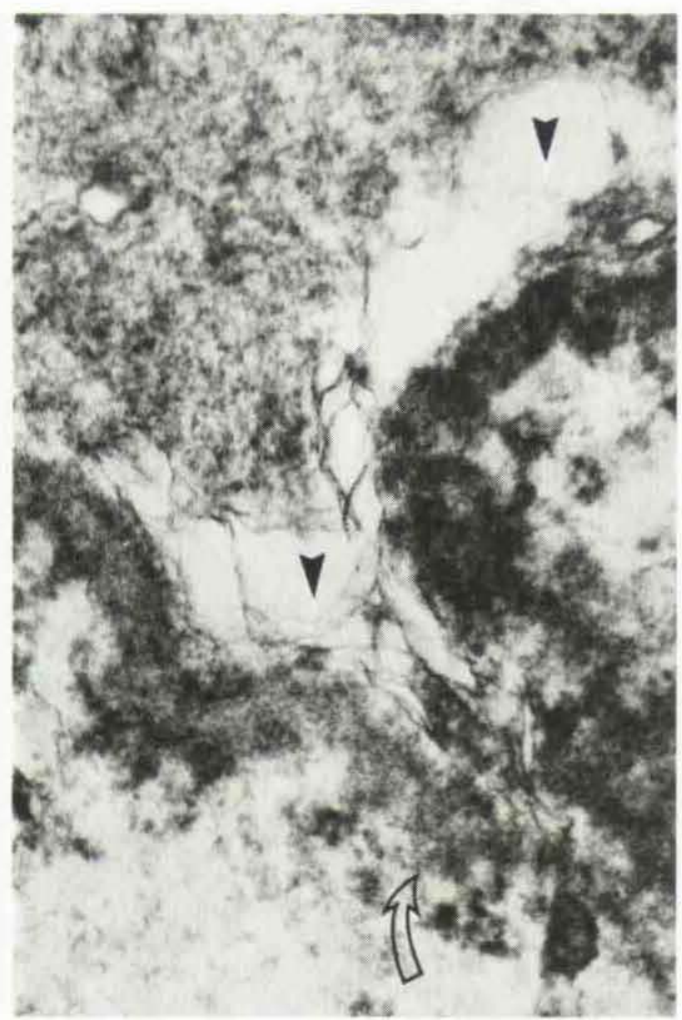

Figure 3. Higher magnification of Fig 2. Open arrows identify lamina densa and sub-lamina densa tissue-bound immunoreactants and arrowheads demonstrate marked focal separation within the level of the lamina lucida. (magnification: $82,500 x)$.

The authors gratefully acknowledge the excellent technical assistance of Mrs. Barbara Stewart and Mr. Ron Austin during the course of this study.

\section{REFERENCES}

1. Spiers EM, Fine JD: Immunopathogenesis of bullous diseases. In: Demis DJ (ed.). Clinical Dermatology. Harper \& Row, Philadelphia, 1987, Unit 6-1, pp. 1-29

2. Gammon WR, Briggaman RA, Woodley DT, Heald P, Wheeler CE Jr: Epidermolysis bullosa acquisita - a pemphigoid-like disease. J Am Acad Dermatol 11:820 - 832, 1984

3. Yaoita H, Katz SI: Immunoclectronmicroscopic localization of IgA in skin of patients with dermatitis herpetiformis. J Invest Dermatol 67:502-506, 1976

4. Stingl G, Honigsmann $\mathrm{H}$, Holubar K, Wolff K: Ultrastructural localization of immunoglobulins in dermatitis herpetiformis. J Invest Dermatol 67:507-512,1976

5. Yaoita $\mathrm{H}$ : Identification of $\mathrm{IgA}$ binding structures in skin of patients with dermatitis herpetiformis. J Invest Dermatol 71:213-216, 1978

6. Klein GF, Hintner H, Schuler G, Fritsch P: Junctional blisters in acquired disorders of the dermal-epidermal junction zone: role of the lamina lucida as the mechanical locus minoris resistentia. $\mathrm{Br} J$ Dermatol 109:499-508, 1983

7. Karttunen T, Autio-Harmainen H, Rasanen O, Risteli J, Risteli L: Immunohistochemical localization of epidermal basement mem- brane laminin and type IV collagen in bullous lesions of dermatitis herpetiformis. Br J Dermatol 111:389-394, 1984

8. Briggaman RA, Gammon WR, Woodley DT: Epidermolysis bullosa acquisita of the immunopathological type (dermolytic pemphigoid). J Invest Dermatol 85:79s - 84s, 1985

9. Nieboer C, Boorsma DM, Woerdeman MJ, Kalsbeek GL: Epidermolysis bullosa acquisita: immunofluorescence, electron microscopic and immunoelectron microscopic studies in four patients. Br J Dermatol 102:383-392, 1980

10. Yaoita H, Briggaman RA, Lawley TJ, Provost TT, Katz SI: Epidermolysis bullosa acquisita: ultrastructural and immunological studies. J Invest Dermatol 76:288 - 292, 1981

11. Gammon WR, Briggaman RA, Inman AO III, Queen LL, Wheeler CE: Differentiating anti-lamina lucida and anti-sublamina densa anti-BMZ antibodies by indirect immunofluorescence on $1.0 \mathrm{M}$ sodium chloride-separated skin. J Invest Dermatol 82:139-144, 1984

12. Hintner H, Stingl G, Schuler G, Fritsch P, Stanley J, Katz S, Wollf K: Immunofluorescence mapping of antigenic determinants within the dermal-epidermal junction in mechanobullous diseases. J Invest Dermatol 76:119-124, 1981

13. Fine JD, Hintner H, Katz SI: Immunofluorescence studies in epidermolysis bullosa utilizing polyclonal and monoclonal antibodies. In: Beutner EH, Chorzelski TP, Kumar V (eds.). Immunopathology of the Skin, 3rd ed. John Wiley \& Sons, Inc., New York, 1987, pp 399-405

14. Yaoita H, Gullino M, Katz SI: Herpes gestationis. Ultrastructure and ultrastructural localization of in vivo- bound complement. Modified tissue preparation and processing for horseradish peroxidase staining of skin. J Invest Dermatol 66:383 -388, 1978

15. Fine JD, Neises GR, Katz SI: Immunofluorescence and immunoelectron microscopic studies in cicatricial pemphigoid. J Invest Dermatol $82: 39-43,1984$

16. Katz SI: The epidermal basement membrane zone - structure, ontog eny, and role in disease. J Am Acad Dermatol 11:1025-1037, 1984

17. Fine JD: The skin basement membrane zone. Adv Dermatol 2:283 304,1987

18. Hall RP, Lawley TJ, Smith HR, Katz SI: Bullous eruption of systemic lupus erythematosus: dramatic response to dapsone therapy. Ann Intern Med 97:165-170, 1982

19. Olansky AJ, Briggaman RA, Gammon WR, Kelly TF, Sams WM Jr: Bullous systemic lupus erythematosus. J Am Acad Dermatol 7:511 520,1982

20. Barton DD, Fine JD, Gammon WR, Sams WM Jr: Bullous systemic lupus erythematosus: an unusual clinical course and detectable circulating autoantibodies to the epidermolysis bullosa acquisita antigen. J Am Acad Dermatol 15:369 - 373, 1986

21. Wilson BD, Birnkrant AF, Beutner EH, Maize JC: Epidermolysis bullosa acquisita: a clinical disorder of varied etiologies. Two cases and a review of immunologic and other reported findings. J Am Acad Dermatol 3:280-291, 1980

22. Briggaman RA, Schechter NM, Fraki J, Lazarus GS: Degradation of the epidermal-dermal junction by proteolytic enzymes from human skin and human polymorphonuclear leukocytes. J Exp Med 160:1027-1042, 1984

23. Gammon WR, Inman AO III, Wheeler CE Jr: Differences in complement-dependent chemotactic activity generated by bullous pemphigoid and epidermolysis bullosa acquisita immune complexes: demonstration by leukocytic attachment and organ culture methods. J Invest Dermatol 83:57-61, 1984

24. Gammon WR: Bullous skin diseases. In: Stone J (ed.). Dermatologic Immunology and Allergy. CV Mosby, St. Louis, 1984, pp 457-493

25. Horiguchi Y, Imamura S: Discrepancy between the localization of in vivo bound immunoglobulins in the skin and in vitro binding sites of circulating anti-BMZ antibodies in bullous pemphigoid: immunoelectron microscopic studies. J Invest Dermatol 87:715-719, 1986 